\title{
Platelet and Transforming Growth Factor Beta 1 Concentrations in Platelet-rich Plasma in Dogs
}

\author{
Inmaculada Peris ${ }^{1}$, Blanca Esquembre ${ }^{1, ~ *}$, Jose Ignacio Redondo ${ }^{2}$, Vicente Jose Sifre ${ }^{1}$, Carme Soler ${ }^{1}$, \\ Claudio Ivan Serra ${ }^{1}$ \\ ${ }^{1}$ Department of Animal Medicine and Surgery, Faculty of Veterinary and Experimental Sciences, Catholic University of Valencia "San Vicente \\ Martir", Valencia, Spain \\ ${ }^{2}$ Faculty of Veterinary, CEU - Cardenal Herrera University, CEU Universities, Valencia, Spain
}

\section{Email address:}

Inmaperis83@gmail.com (I. Peris), blanca.esquembre@gmail.com (B. Esquembre), nacho@uchceu.es (J. I. Redondo),

vj.sifre@ucv.es (V. J. Sifre),mdc.soler@ucv.es (C. Soler), ci.serra@ucv.es (C. I. Serra)

${ }^{*}$ Corresponding author

\section{To cite this article:}

Inmaculada Peris, Blanca Esquembre, Jose Ignacio Redondo, Vicente Jose Sifre, Carme Soler, Claudio Ivan Serra. Platelet and Transforming Growth Factor Beta 1 Concentrations in Platelet-rich Plasma in Dogs. Animal and Veterinary Sciences. Vol. 8, No. 4, 2020 , pp. 93-100. doi: $10.11648 /$ j.avs.20200804.15

Received: July 20, 2020; Accepted: August 5, 2020; Published: August 19, 2020

\begin{abstract}
Platelet-rich plasma showed good results in tissue healing when first used in human medicine. After that, its use spread to veterinary medicine. However, there is no standardized method for manual collection of platelet - rich plasma in the canine species. The objectives of this study were to standardize a protocol to obtain platelet - rich plasma (PRP) with high concentration of platelets and transforming growth factor $\beta 1$ (TGF - $\beta 1$ ) without the presence of erythrocytes and leukocytes; and to relate the presence of TGF - $\beta 1$ with the amount of platelets. For this purpose, there were obtained two blood samples separated one week between each other, from eleven healthy Beagles. Blood samples were centrifuged using different protocols: protocol A (one centrifuge, $210 \mathrm{~g}$ and 10 minutes), protocol B (double centrifuge, first one $210 \mathrm{~g}$ and 10 minutes, second one 210 $\mathrm{g}$ and 15 minutes) and protocol $\mathrm{C}$ (one centrifuge, $475 \mathrm{~g}$ and 8 minutes). Three plasma fractions were obtained through these protocols: a platelet-rich fraction, a platelet-poor fraction and whole blood. The content of leukocytes, erythrocytes and platelets was measured in the whole blood and plasma rich and poor fractions of the protocol A, B and C. The TGF - $\beta 1$ concentration was measured in the platelet rich and poor fraction of A and B protocols. The results showed a higher concentration of platelets and TGF $-\beta 1$ in protocol A. In conclusion, this study offers an economical and reproducible method for obtaining PRP in the canine specie.
\end{abstract}

Keywords: Dog, Platelet, PRP, Growth Factor, TGF $-\beta 1$

\section{Introduction}

Platelet - rich plasma (PRP) is an autogenous fluid concentrate composed primarily of platelets and growth factors. Platelets play roles in both haemostasis and wound healing, as well as contain granules that release growth factors to stimulate other cells of the body to migrate to the area of the trauma, thus facilitating tissue healing. These growth factors include platelet - derived growth factor (PDGF), transforming growth factor $\beta 1$ and $\beta 2$ (TGF $-\beta 1$, TGF $-\beta 2$ ), vascular endothelial growth factor (VEGF), basic fibroblast growth factor (bFGF) and epidermal growth factor (EGF) [1].
In the $1990 \mathrm{~s}$, several authors began to use PRP for oral and maxillofacial grafts in human medicine [2]. Since this discovery, the use of these types of preparations rich in growth factors has been extrapolated to different fields of regenerative medicine. The objective is to achieve faster, more effective and less aggressive recovery therapies, and to improve the quality of life of the patient [3]. At the same time that these techniques were being developed in human medicine; the veterinary sector, especially the discipline of orthopaedics and traumatology, has begun to incorporate platelet rich plasma (PRP) along with other therapeutic tools $[4,5]$. In fact, some authors showed promising results of the PRP used in wound healing, osteoarthritis or improving functional outcome after 
surgery repair for cranial cruciate ligament in dogs [6 - 9].

In human medicine, there is a wide range of commercial preparations rich in growth factors, as well as different kits to obtain these mixtures in an automatic or manual way [10]. On the other hand, in the veterinary field these kits are rare in the commercial form, expensive and have inconsistent cell content. The majority of the veterinary available kits have different cell types besides the platelets (red blood cells, white blood cells). Furthermore, protocols for obtaining this autologous plasma in the canine species have been extrapolated from human medicine, without taking into account the hematological differences between species, such as the lower mean corpuscular volume and mean corpuscular haemoglobin in dogs; which means that a relatively higher centrifugal force is required to separate canine blood components [1, 10-12].

For this reason, the main objective of this study was to standardize an effective and easily reproducible method to obtain PRP in the canine species in which platelet concentrations are approximately 2-3 times higher than the concentration in peripheral blood, without the presence of red and white blood cells. In addition, it was established as a secondary objective to relate the presence of TGF- $\beta 1$ with the amount of platelets obtained, and for that, the protocol that more platelets obtained was compared with the protocol that obtained less platelets.

\section{Material and Methods}

\subsection{Animals and Husbandry}

11 Beagle dogs (Canis familiaris) were enrolled in the study. The animals included were 6 intact males and 5 intact females with a median age of 29.18 months ( 15 months -66 months) and a median weight of $14.17 \mathrm{~kg}(9-22.8 \mathrm{~kg})$. The inclusion criteria stipulated that the animals needed to be in adequate health, which was determined by a general physical exam, hematology, biochemistry and serological testing to exclude Leishmania infantum, Leishmania donovani, Dirofilaria immitis, Borrelia Burgdorferi and Ehrlichia canis.

The animals were housed in individual cages in a climate controlled room at $24 \pm 2{ }^{\circ} \mathrm{C}, 60-70 \%$ relative humidity and 12 hours of light from 07:00 to 19:00 (photophase). There were fed with a commercial dog standard diet following the recommendations of the brand according to their weight (Royal Canin Medium Adult Dog, Royal Canin, Madrid, Spain), and had ad libitum water access.

The present study was approved by the local authorities according to the Directive 2010/63/EU of the European Parliament on the protection of animals used for scientific purposes; and to the local animal welfare legislation on the protection of animals used for experimental and other scientific purposes.

\subsection{Experimental Technique}

Blood sampling was performed to the eleven dogs on two occasions, one week apart, because it was not feasible to obtain the large blood sample required to measure the platelet, red blood cell, leukocyte and TGF- $\beta 1$ concentrations. The first sample was taken to obtain the quantity of platelets, white blood cells and red blood cells; and the second one to obtain the amount of TGF- $\beta 1$.

The first blood samples were obtained from the jugular vein. There were extracted 16 millilitres, which were divided in several tubes with different anticoagulant agents. Three sodium citrate tubes (BD Vacutainer ${ }^{\circledR}$ 9NC, BD-Plymouth, Plymouth, United Kingdom) of $4.5 \mathrm{ml}$ each one were collected. One of the sample tubes was used to do a hematology, made by a hematology analyser (Celltac Alpha MEK-6318 J/K, NIHON KOHDEN, Tokyo, Japan), and the other ones were used to obtain the plasma by centrifuging. A separate heparin tube (LH/Li Heparin, AQUISEL ${ }^{\circledR}$, Barcelona, Spain) of $2 \mathrm{ml}$ was collected to do a biochemistry profile (METROLAB 2300, Metrolab, Buenos Aires, Argentina), and a serological testing made by different commercial kits: Canine Leishmania Antibody Test Kit (SNAP Leishmania, IDEXX LABORATORIES, Barcelona, Spain) and Canine Heartworm Antigen/Borrelia Burgdorferi/Ehrlichia canis Antibody Test Kit (SNAP 3DX, IDEXX Laboratories, Barcelona, Spain).

The second sampling was performed 7 days after the first one. Nine millilitres of blood were extracted from the jugular vein and divided in two sodium citrate tubes of $4.5 \mathrm{ml}$ (BD Vacutainer $^{\circledR}$ 9NC, BD-Plymouth, Plymouth, United Kingdom). These samples were used to quantify TGF- $\beta 1$ in the plasmatic fractions after application of different centrifuge protocols.

\subsubsection{Obtaining the Plasmatic Fraction}

In every centrifuge protocol and for all the tests performed, the plasmatic fraction was obtained in a standardised way. All the blood samples from the eleven dogs underwent the three different centrifuge protocols. The centrifuge was a swinging head centrifuge (CENTRONIC P Selecta, J. P Selecta, Barcelona, Spain). The centrifuge protocols are detailed in Table 1. Protocol A involved a single centrifuge at $210 \mathrm{~g}$ relative centrifuge force for 10 minutes (proposed protocol). Protocol B involved two consecutive centrifuges. At the first centrifuge $210 \mathrm{~g}$ relative centrifuge force for 10 minutes was performed. After the plasma was separated, it underwent a second centrifuge using $210 \mathrm{~g}$ relative centrifuge force for a further 15 minutes $[13,14]$. Finally, protocol $\mathrm{C}$ involved a single $475 \mathrm{~g}$ relative centrifuge force during 8 minutes [15].

Once the blood was centrifuged, the plasma remained on the top part of the tube and the "pellet" where the erythrocytes were located, was at the bottom of the tube. In between these two zones, above the fraction with the red blood cells, a whitish layer (buffy coat) can be seen; this layer was where all the leukocytes were located.

From the plasmatic portion, $200 \mu \mathrm{l}$ was extracted from the top part of the tube; this part is to be considered the platelet poor plasma (PP), the last $200 \mu \mathrm{l}$, which are closer to the buffy coat is considered the platelet rich plasma (PR).

In the protocol where two centrifuges were performed, after 
the first centrifuge, the plasmatic portion was taken in its entirety and placed separately in a sterile container that was centrifuged again. After this second centrifuge, the respective portions of PP and PR were extracted in the same way (the first $200 \mu \mathrm{l}$ was deemed the PP and the second $200 \mu$ obtained closer to the pellet was deemed the PR).

Table 1. Centrifuge protocols.

\begin{tabular}{lllllll}
\hline \multirow{2}{*}{ Protocol } & A & & B & & C \\
\cline { 2 - 6 } & g & min. & g & min. & g \\
\hline $1^{\text {st }}$ centrifuge & 210 & 10 & - & 210 & 10 & 475 \\
$2^{\text {nd }}$ centrifuge & - & 210 & 15 & - \\
\hline
\end{tabular}

$+\mathrm{g}$ : relative centrifuge force, $\min .:$ minutes.

\subsubsection{Platelet, Leukocyte and Erythrocyte Count Analysis}

As the objective was to understand which centrifuge protocol yields the highest platelet concentration before the TGF- $\beta 1$ quantification, several platelet and leukocyte counts at different time intervals and different relative centrifuge forces were performed by a hematology analyser (Celltac Alpha MEK-6318 J/K, NIHON KOHDEN, Tokyo, Japan).

In the first part of the study, the platelet, the leukocyte and the erythrocyte concentration were analysed in the central blood stream, the platelet poor plasma (PP) fraction and the platelet rich plasma $(\mathrm{PR})$ fraction.

\subsubsection{Transforming Growth Factor Beta - 1}

To quantify levels of TGF- $\beta 1$ in plasma the commercial kit (Quantikine $^{\circledR}$ ELISA kit, RD Systems, Abingdon, United Kingdom) was used. This kit's technique is based on an enzymatic reaction: ELISA (Enzyme-Linked ImmunoSorbent Assay).

The TGF- $\beta 1$ was measured in the obtained PP and PR under the described centrifuge protocols (protocol A and protocol B). These fractions were obtained using the same methods aforementioned to quantify the platelets and the white blood cells. The samples needed activation that was carried out using a different variety of reagents and diluents that were included in the kit previously prepared.

The automatic microplaque lector used was the Personal lab junior (Adaltis Vitro S. A, Milano, Italy). This machine uses a computer program that calculates and creates the standard curve automatically (WordBench-V2.3).

\subsection{Statistic Study}

The formula used to calculate the required sample size was the one that is used to compare two different measures. This study considers a $99 \%$ security and a $95 \%$ statistic power; therefore, coefficients $Z_{\alpha}$ and $Z_{\beta}$ will be 2.57 and 1.64 respectively. For "d" a value of $50 \%$ was taken. To obtain the security and the statistic power described, the correct sample size was 9.2 dogs. However, as the study used individual animals, 10 dogs were described as (n).

Once the numeric values for each one of the samples in our study was described, the average was calculated (x), as was the standard deviation (SD) and the minimum and maximum value for each variable and centrifuge protocol. To obtain the level of statistic significance, a variance analysis was performed, one way ANOVA; using the platelet, white blood cells and TGF- $\beta 1$ concentrations as a dependent variables. The centrifuge protocols and plasmatic fractions were described as independent variables. The statistical decisions were made using $\mathrm{p}<0.05$. The informatics treatment and the statistical analysis of the obtained data were processed with Statistica for Windows ${ }^{\circledR}$ version 6.0 (StatSoft Inc.; USA).

\section{Results}

All the animals were healthy and were included in the present study (11/11). There were no adverse events in any animal after performing blood sampling.

Once the measurements and deviations from all variables were obtained, these results were compared within the three different protocols (Table 2).

In Figure 1, the comparison of the platelet variable is shown. The PR (platelet rich plasma) fraction was significantly higher in protocol A, followed by protocol C, with the lowest platelet concentration found in protocol B. Comparing the different plasmatic fractions within each protocol, in protocol A the PR concentration was significantly higher when compared to blood and PP; and between the latter no significant differences were noted. Protocol B showed no differences between PP and PR; and both of them were significantly lower than blood. Finally, in protocol C, PR and blood fractions had no differences, but were significantly higher than PP.

In Figure 2, the comparative study of the white cell variable is described. In the analysis of plasmatic fractions, the results were the same for the three different fractions: there were no significant differences between protocols. During the comparison between fractions within the same protocol, the blood turned out to be the highest and significantly different to the PP and PR, between which there was no difference and trended towards 0 leukocyte/ $\mu$ l. The same results were obtained regarding red blood cells.

Figure 3 shows the comparative study of the TGF $-\beta 1$ variable in $\mathrm{A}$ and $\mathrm{B}$ protocols. There were no significant differences between the PP and PR fractions of both protocols. However, in protocol A, TGF - $\beta 1$ in the PR fraction was significantly higher compared to PP fraction, while there were no significant differences between the PR and PP fractions of protocol B. 


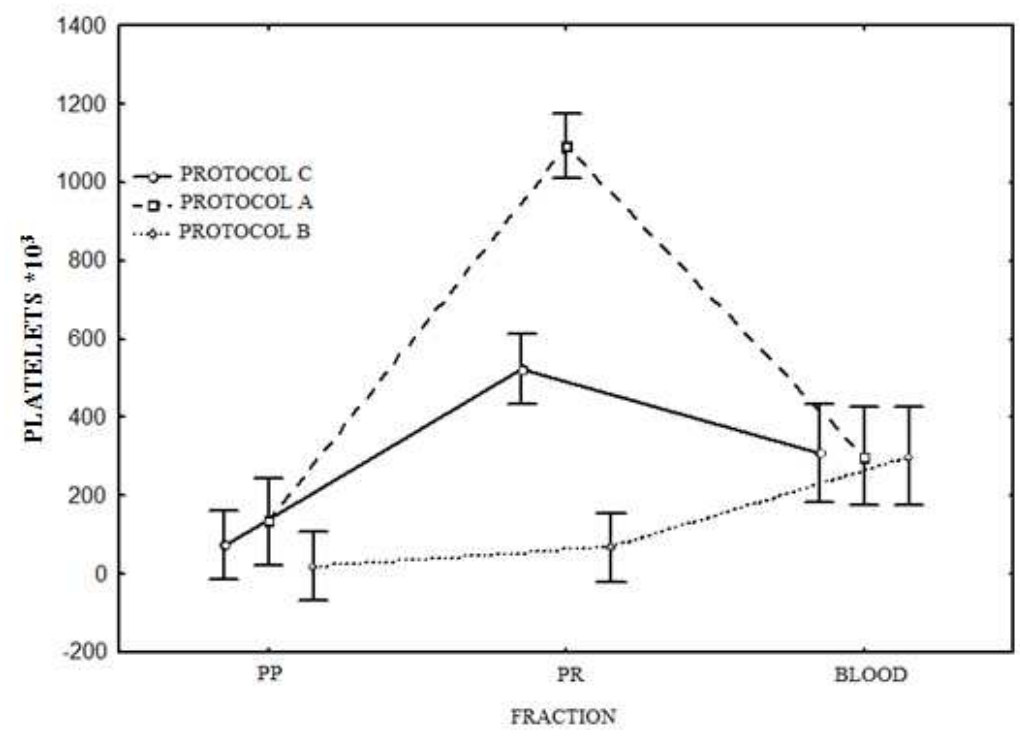

Figure 1. Comparative study of the platelet variable.

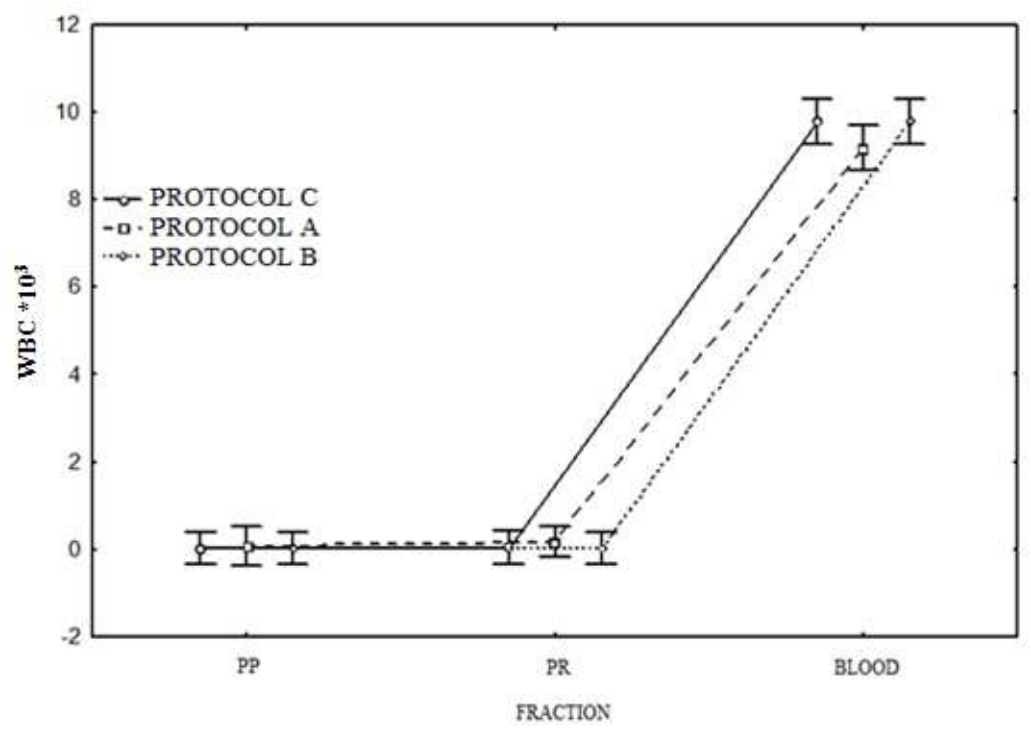

Figure 2. Comparative study of the white blood cells variable.

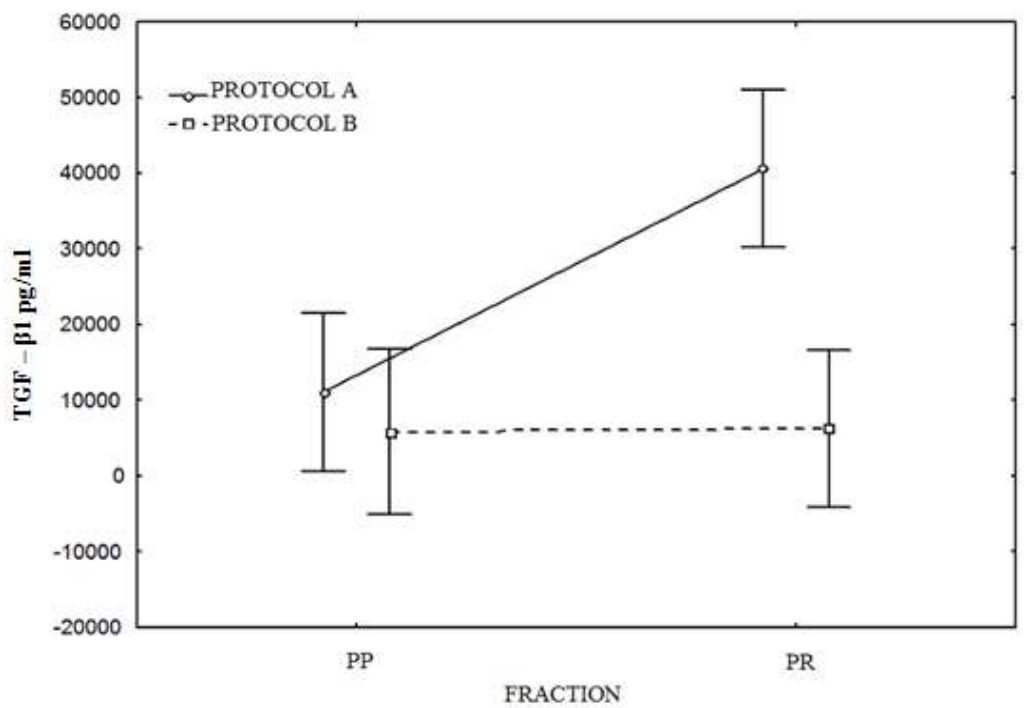

Figure 3. Comparative study of the TGF- $\beta 1$ variable. 
Table 2. Platelet, leukocyte and TGF- $\beta 1$ concentrations in the different protocols.

\begin{tabular}{|c|c|c|c|c|c|c|}
\hline PRT. & FRC. & VARIABLE & $\mathbf{X}$ & SD & MIN & MAX \\
\hline \multirow{8}{*}{ A } & \multirow{3}{*}{ PP } & PLT & 128 & 60 & 66 & 239 \\
\hline & & WBC & 0.1 & 0.1 & 0 & 0.4 \\
\hline & & TGF- $\beta 1$ & 11054.0 & 14426.6 & 3672 & 52835.7 \\
\hline & \multirow{3}{*}{ PR } & PLT & 1013 & 431 & 611 & 1884 \\
\hline & & WBC & 0.1 & 0.1 & 0.0 & 0.3 \\
\hline & & TGF- $\beta 1$ & 40614.6 & 3025.4 & 7465.8 & 106310.5 \\
\hline & \multirow{3}{*}{ BLOOD } & PLT & 301 & 53 & 239 & 377 \\
\hline & & WBC & 9.2 & 1.9 & 5.7 & 11.9 \\
\hline \multirow{8}{*}{ B } & & PLT & 22 & 21 & 5 & 77 \\
\hline & \multirow[t]{2}{*}{ PP } & WBC & 0.0 & 0.0 & 0.0 & 0.1 \\
\hline & & TGF- $\beta 1$ & 5807.3 & 2783.3 & 2332.8 & 9698.2 \\
\hline & \multirow{3}{*}{ PR } & PLT & 81 & 111 & 9 & 362 \\
\hline & & WBC & 0.0 & 0.0 & 0.0 & 0.1 \\
\hline & & TGF- $\beta 1$ & 6243 & 3639.6 & 2900 & 13192.7 \\
\hline & \multirow{2}{*}{ BLOOD } & PLT & 300 & 67 & 226 & 408 \\
\hline & & WBC & 9.8 & 1.9 & 5.9 & 12.1 \\
\hline \multirow{6}{*}{$\mathrm{C}$} & \multirow{2}{*}{ PP } & PLT & 75 & 31 & 36 & 132 \\
\hline & & WBC & 0.0 & 0.0 & 0.0 & 0.1 \\
\hline & \multirow{2}{*}{ PR } & PLT & 516 & 370 & 178 & 1477 \\
\hline & & WBC & 0.0 & 0.0 & 0.0 & 0.1 \\
\hline & \multirow{2}{*}{ BLOOD } & PLT & 307 & 61 & 226 & 408 \\
\hline & & WBC & 9.8 & 1.9 & 5.9 & 12.1 \\
\hline
\end{tabular}

+PRT: protocol, FRC: fraction, X: media, SD: standard deviation, MIN: minimum value, MAX: maximum value.

+PP: platelet poor plasma, PR: platelet rich plasma, PLT: platelet, WBC: white blood cells, TGF- $\beta 1$ : transforming growth factor beta 1 .

+ PLT and WBC are expressed in $10^{3} \mathrm{cel} / \mu 1$ and TGF- $\beta 1$ in $\mathrm{pg} / \mathrm{ml}$.

\section{Discussion}

\subsection{Method and Study Design}

The canine specie was chosen as a model due to easy handling and kennelling, and clinical benefit. This also offers a standardised method for further usage as a model in further experimental studies and can be extrapolated to direct clinical application.

There are two main methods to obtain PRP, via manual method $[13,14,16-18]$ or via commercially available kits $[15,19-21]$. In this study, manual methods were used, as the objective was to find a practical utility, which could be used easily in "in vivo" studies and general practice. Besides, PRPs obtained by commercially available systems can potentially differ in their characteristics. Two previous studies that compared different commercially available kits saw that results differed for a number of factors, including the degree of platelet concentration, leukocyte concentration or the presence of erythrocytes $[22,1]$. Additionally, given the difficulty sourcing commercial kits, and their expense, the manual method of platelet extraction is a valid alternative in this species; as long as aseptic and sterile conditions are maintained.

Regarding the centrifuge protocol, literature describes the method of one versus several centrifuges (usually two) [13, $14,16,18,19]$. Most of the literature shows that a double centrifuge method achieves higher platelet concentrations. However, this method can cause alterations in platelet morphology and is more sensitive to small processing errors
[23]. Other studies showed that a single centrifuge can produce comparable amounts of platelets for their therapeutic applications [24]. In the present study, it was considered that by performing a single centrifuge, preparation time is reduced and contamination risk is minimised.

The two previously described chosen protocols for this study, were selected as the relative centrifuge force units were described in "g", the results obtained were referenced at least for the platelet concentrations and the manual method was employed [13 - 15].

There were three protocols proposed in this study. Regarding human medicine literature, the best results for platelet recovery were obtained with a relative centrifuge force of 200-300 g and time ranges from five to twelve minutes, with a simple or double centrifuge. That is why the authors chose protocol A (single centrifuge, $210 \mathrm{~g}$ for 10 minutes) and protocol B (double centrifuge, $210 \mathrm{~g}$, for 10 and 15 minutes). However, other published protocols obtained a high platelet concentration using higher relative centrifuge forces. Therefore, the authors proposed protocol C (single centrifuge, $475 \mathrm{~g}$ for 8 minutes) $[1,12,25-28]$.

The qualitative and quantitative evaluation for the growth factor TGF- $\beta 1$ in this study was chosen because it is one of the more thoroughly studied growth factors [29]. It is a cytokine involved in tissue reparation and regeneration processes, and tends to be the most frequently chosen cytokine for quantification in plasma rich in growth factor studies [21].

\subsection{Results}

On the basis of the platelet results obtained in each protocol, 
protocol A satisfies the proposed objective of obtaining statistically higher platelet concentrations in platelet rich plasma (PRP), obtaining an average value in the PRP of 1013 x $10^{3}$ platelets $/ \mu 1$. This significantly high value achieves a 2.36 platelet ratio, which corresponds to the published literature that has obtained positive results in its therapeutic application. Moreover, is a value superior to $1000 \times 10^{3}$ platelets/ $\mu 1$, which is the minimum value referred by some authors to obtain beneficial results over the tissue repair and regeneration process [2, $30-32]$. Other authors discussed that a platelet amount superior to $200-300 \times 10^{3}$ platelets/ $\mu$ l has a therapeutic effect $[12,24]$.

The results obtained with protocol B were unexpected, as other authors showed a higher platelet concentration with a double centrifuge method. This might be explained by the fact that the platelet rich fraction remained trapped in the sediment that appeared after the second centrifuge; while the authors referenced could have re-suspended the platelet rich fraction of the sediment, obtaining a higher platelet rich plasma fraction $[13,14]$.

Finally, protocol $\mathrm{C}$ showed higher platelet values than protocol B but lower than protocol A. The authors think that the higher relative centrifuge force could have driven a high number of platelets to the buffy coat fraction, with most of the white blood cells.

Of the three described protocols, white blood and red blood cell concentrations were very similar on their PP and PR fractions, without significant differences, and statistically lower than that of peripheral blood. Furthermore, the absolute value of these plasmatic fractions was around 0 leukocytes $/ \mu 1$ achieving (in the three protocols) one of the goals proposed for the PRP fraction in this study [3]. It is thought that high leukocyte concentrations in PRP can impede tissue regeneration and healing by increasing the amount of pro inflammatory cytokines and thereby raising the acute inflammatory response, although it remains a matter of disagreement in the literature $[1,22]$. There were obtained the same results regarding erythrocyte concentration, with absolute values trending towards 0 erythrocytes $/ \mu$. Erythrocytes can cause cell death and increase the presence of inflammatory mediators, so only PRPs with a negligible amount of erythrocytes should be used for therapeutic applications $[1,22]$.

Concerning the platelet ratio, protocol A can be compared to some of the tested commercial systems. Protocol A obtained a platelet ratio of 2.36, whereas some commercial systems only obtained 0.85 or 1.84 . Other commercial systems tested showed a higher platelet ratio (3.21, 5.15 and 3.98), but also a higher leukocyte ratio $(0.17,3.27$ and 1.40 respectively). The haematocrit was also measured in those systems, and the systems with the highest platelet ratio showed a haematocrit greater than zero [22].

In relation to the TGF- $\beta 1$ concentration, protocol $A$ and protocol $\mathrm{B}$ were compared to assess the correlation between the amount of platelets and the presence of this growth factor. Ideally, the study would have considered the three protocols, but unfortunately the authors were unable due to economical reasons.

Regarding the comparison between protocols, significantly higher values in cytokine concentration in the PRP for protocol A was observed when compared to protocol B. Following various author references who highlighted the presence of a polypeptide substance inside the $\alpha$ granules from the platelets, which is released after the cellular activation process [33], the results are not surprising when differences in platelet concentrations between groups are observed. Moreover, some authors confirmed a strong correlation between the TGF- $\beta 1$ and platelet concentrations, or the amount of TGF- $\beta 1$ and the number of activated platelets $[12$, 34].

Results also showed that there were no significant differences when comparing TGF- $\beta 1$ concentrations in the PP between protocols (as it was seen in the platelet concentration). This supports the hypothesis of other studies that describe the importance of the presence of this growth factor in its free form in plasma in addition to its intracellular form, as well as the influence in size and platelet age in the concentration of $\alpha$ granules [35].

It needs to be considered that there were no differences between PR and PP inside each protocol. This is due to the high standard deviation (SD) in the TGF- $\beta 1$ values in respect to the mean $(\mathrm{X})$; that confirms the existing high variability of the growth factor concentration between individuals of the same species [13, 16, 21].

This study had an economical limitation that impeded the authors to compare the amount of TGF - $\beta 1$ between the three proposed protocols or to evaluate other growth factors. However, the objective of this study was just to evaluate the concentration of platelets and its relation with the amount of TGF - $\beta 1$, what was achieved by comparing protocol A (which had the highest number of platelets) with protocol B (which had the lowest number of platelets).

It also can be a limitation the fact that TGF- $\beta 1$ was also present in protocol $\mathrm{B}$ and no statistical differences were found between protocols, what could be explained by the presence of TGF- $\beta 1$ in a free plasmatic form. For this reason, it would be probably better to measure another growth factor present only inside $\alpha$ granules of the platelets instead the TGF- $\beta 1$.

It must be taken into account that it has not been established yet a standard concentration of platelets on the use of PRP in dogs. It remains unknown the response of different canine cell types (chondrocytes, myocytes, chondrocytes, etc.) to various platelet or growth factor concentrations, and it has to be remembered that not the highest platelet concentration is necessarily the best [22].

\section{Conclusion}

From the obtained results and under the study conditions previously explained, we can conclude that the proposed protocol in this study (protocol A: $210 \mathrm{~g}$ for 10 minutes) should be the chosen protocol for clinical use in the canine species. We conclude that with a simple, economical and reproducible methodology; plasmatic fractions (especially 
the PR fraction) with a high concentration of platelets and TGF- $\beta 1$ can be obtained, without the presence of white or red blood cells.

\section{Acknowledgements}

This work was supported by "Glamour Journal Grant" for young researchers.

\section{References}

[1] Carr BJ, Canapp Jr SO, Mason DR, Cox T, Hess T. Canine Platelet-Rich Plasma Systems: A Prospective Analysis. Front Vet Sci 2016; 2: 73.

[2] Marx RE, Carlson ER, Eichstaedt RM, Schimmel SR, Strauss JE, Georgeff KR. Platelet-rich plasma: Growth factor enhancement for bone grafts. Oral Surg Oral Med Oral Pathol Oral Radiol Endod 1998; 85 (6): 638-46.

[3] Anitua E, Sánchez M, Orive G, Andía I. The potential impact of the preparation rich in growth factors (PRGF) in different medical fields. Biomaterials 2007; 28 (31): 4551-60.

[4] Karayannopoulou M, Papazoglou LG, Loukopoulos P, et al. Locally injected autologous platelet-rich plasma enhanced tissue perfusion and improved survival of long subdermal plexus skin flaps in dogs. Vet Comp Orthop Traumatol 2014; 4; 27 (5).

[5] Rabillard M, Grand J-G, Delibert E, Fellah B, Gauthier O, Niebauer GW. Effect of autologous platelet rich plasma gel and calcium phosphate biomaterials on bone healing in an ulnar ostectomy model in dogs. Vet Comp Orthop Traumatol 2009; 22 (6): 460-6.

[6] Jee CH, Eom NY, Jang HM, et al. Effect of autologous platelet-rich plasma application on cutaneous wound healing in dogs. J Vet Sci 2016; 17 (1): 79-87.

[7] Fahie MA, Ortolano GA, Guercio V, et al. A randomized controlled trial of the efficacy of autologous platelet therapy for the treatment of osteoarthritis in dogs. JAVMA 2013; 243 (9): 1291-1297.

[8] Hilty DH, Yocum JG Y, Gallichio VS. Examining the Effectiveness of Stem Cell and Platelet-Rich Plasma Therapies for Treating Osteoarthritis in Dogs. Anim Husb Dairy Vet Sci 2017; 1 (4).

[9] Silva RF, Carmona JU, Rezende CMF. Intra-articular injections of autologous platelet concentrates in dogs with surgical reparation of cranial cruciate ligament rupture. Veterinary and Comparative Orthopaedics and Traumatology 2013; 26 (4): 285-290.

[10] Magalon J, Bausset O, Serratrice N, et al. Characterization and comparison of 5 platelet-rich plasma preparations in a single-donor model. Arthroscopy 2014; 30 (5): 629-638.

[11] Stief M, Gottschalk J, Ionita J-C, Einspanier A, Oechtering G, Böttcher P. Concentration of platelets and growth factors in canine autologous conditioned plasma. Vet Comp Orthop Traumatol 2011; 24 (2): 122-5.

[12] Shin HS, Woo HM, Kang BJ. Optimisation of a double-centrifugation method for preparation of canine platelet-rich plasma. BMC Vet Res 2017; 13 (1): 198.

[13] Casati MZ, De Vasconcelos Gurgel BC, Gonçalves PF, et al. Platelet-rich plasma does not improve bone regeneration around peri-implant bone defects--a pilot study in dogs. Int J Oral Maxillofac Surg 2007; 36 (2): 132-6.

[14] De Vasconcelos Gurgel BC, Goncalves PF, Pimentel SP, et al. Platelet-rich plasma may not provide any additional effect when associated with guided bone regeneration around dental implants in dogs. Clin Oral Implants Res 2007; 18 (5): 649-54.

[15] Anitua E, Andia I, Ardanza B, Nurden P, Nurden AT. Autologous platelets as a source of proteins for healing and tissue regeneration. Throm Haemost 2004; 91 (1): 4-15.

[16] Okuda K, Kawase T, Momose M, et al. Platelet-rich plasma contains high levels of platelet-derived growth factor and transforming growth factor-beta and modulates the proliferation of periodontally related cells in vitro. J Periodontol 2003; 74 (6): 849-57.

[17] Ouyang XY and Qiao J. Effect of platelet-rich plasma in the treatment of periodontal intrabony defects in humans. Chin Med J (Engl) 2006; 119 (18): 1511-21.

[18] Carmona JU, Argüelles D, Climent F, Prades M. Autologous platelet concentrates as a treatment of horses with osteoarthritis: A preliminary pilot clinical study. Journal of Equine Veterinary Science 2007; 27: 167-170.

[19] Grageda E, Lozada JL, Boyne PJ, Caplanis N, McMillian PJ. Bone formation in the maxillary sinus by using platelet-rich plasma: an experimental study in sheep. J Oral Implantol 2005; 31 (1): 2-17.

[20] Wehling P, Moser C, Frisbie D, et al. Autologous conditioned serum in the treatment of orthopedic diseases: the orthokine therapy. BioDrugs 2007; 21 (5): 323-32.

[21] Weibrich G, Kleis WK, Hitzler WE, Hafner G. Comparison of the platelet concentrate collection system with the plasma-rich-in-growth-factors kit to produce platelet-rich plasma: a technical report. Int J Oral Maxillofac Implants 2005; 20 (1): 118-23.

[22] Franklin SP, Garner BC, Cook JL. Characteristics of canine platelet-rich plasma prepared with five commercially available systems. American journal of veterinary research 2015; 76 (9): 822-827.

[23] Nagata MJ, Messora MR, Furlaneto FA, et al. Effectiveness of two methods for preparation of autologous platelet-rich plasma: an experimental study in rabbits. European journal of dentistry 2010; 4 (4): 395-402.

[24] Mazzocca AD, McCarthy MBR, Chowaniec DM, et al. Platelet-rich plasma differs according to preparation method and human variability. JBJS 2012; 94 (4): 308-316.

[25] Serra CI, Soler C, Carrillo JM, Sopena JJ, Redondo JI, Cugat R. Effect of autologous platelet - rich plasma on the repair of full - thickness articular deffects in rabbit. Knee Surg Sports Traumatol Arthrosc 2013; 21: 1730-1736.

[26] Araki J, Jona M, Eto H, et al. Optimized preparation method of platelet-concentrated plasma and noncoagulating platelet-derived factor concentrates: maximization of platelet concentration and removal of fibrinogen. Tissue Eng Part C Methods 2012; 18 (3): 176-185. 
[27] Amable PR, Carias RBV, Teixeira MVT, et al. Platelet-rich plasma preparation for regenerative medicine: optimization and quantification of cytokines and growth factors. Stem cell research \& therapy 2013; 4 (3): 67.

[28] Monobe MM. Canine pure platelet-rich plasma for regenerative medicine and platelet research: protocol optimization [PhD Thesis]. Meridian, Mississippi: Mississippi State University, 2016.

[29] Chopra R and Anastassiades T. Specificity and synergism of polypeptide growth factors in stimulating the synthesis of proteoglycans and a novel high molecular weight anionic glycoprotein by articular chondrocyte cultures. J Rheumatol 1998; 25 (8): 1578-84.

[30] Gerard D, Carlson ER, Gotcher JE, Jacobs M. Effects of platelet-rich plasma on the healing of autologous bone grafted mandibular defects in dogs. J Oral Maxillofac Surg 2006; 64 (3): 443-51.
[31] Marx RE. The biology of platelet-rich plasma. Journal of Oral Maxillofacial Surgery 2001; 59: 1119-1121.

[32] Weibrich G, Hansen T, Kleis W, Buch R, Hitzler WE. Effect of platelet concentration in platelet-rich plasma on peri-implant bone regeneration. Bone 2004; 34 (4): 665-71.

[33] Gleizes PE, Munger JS, Nunes I, et al. TGF-beta latency: biological significance and mechanisms of activation. Stem Cells 1997; 15 (3): 190-7.

[34] Franklin SP, Birdwhistell KE. Assessment of Canine Autologous Conditioned PlasmaTM Cellular and Transforming Growth Factor- $\beta 1$ Content. Front Vet Sci 2018; 5: 105.

[35] Assoian RK, Komoriya A, Meyers CA, Miller DM, Sporn MB. Transforming growth factor-beta in human platelets. Identification of a major storage site, purification, and characterization. J Biol Chem 1983; 258 (11): 7155-60. 